\title{
Inguinal Lymph Node Squamous Cell Carcinoma of Unknown Primary Site: A Case Report
}

\author{
Pai VD ${ }^{*}$, Kattimani $\mathrm{K}^{2}$, Manohar $\mathrm{V}^{3}$ and Ravindranath $\mathrm{S}^{3}$ \\ ${ }^{1}$ Department of Surgical Oncology, Kerudi Cancer Hospital, Bagalkot 587101, Karnataka, India \\ ${ }^{2}$ Department of Medical Oncology, Kerudi Cancer Hospital, Bagalkot 587101, Karnataka, India \\ ${ }^{3}$ Department of Pathology, SRL Diagnostics, Bangalore 560076, Karnataka, India
}

*Corresponding author: Pai VD, Department of Surgical Oncology, Kerudi Cancer Hospital, Bagalkot 587101, Karnataka, India, Tel: +91-9449333502, E-mail: vishpai88@gmail.com

Citation: Pai VD, Kattimani K, Manohar V, Ravindranath S (2016) Inguinal Lymph Node Squamous Cell Carcinoma of Unknown Primary Site: A Case Report. J Surg Oper Care 1(2): 207. doi: 10.15744/2455-

7617.1.207

Received Date: April 06, 2016 Accepted Date: June 15, 2016 Published Date: June 17, 2016

\begin{abstract}
Carcinoma of unknown primary site (CUP) is a heterogeneous group of malignancies associated with dismal prognosis. Although majority of the patients present with disseminated disease, some of them do present with disease limited to the lymph nodes. Incidence of CUP is decreasing because of the newer diagnostic modalities that are employed in the work up of these patients. Inguinal node squamous cell carcinoma deposits with unknown primary are rare malignancies which are associated with good long term prognosis. No consensus exists for the management of these disorders although surgical resection is advocated by the majority. We are presenting a case of middle aged lady with groin node squamous cell carcinoma deposits with unknown primary who was treated with surgery followed by adjuvant chemotherapy.
\end{abstract}

Keywords: Inguinal node; Squamous cell carcinoma; Unknown primary

\section{Introduction}

Carcinoma of unknown primary site (CUP) accounts for approximately 3\% to 5\% of all cancers [1-3]. However when disseminated, it is one of the leading causes of cancer related mortality worldwide. Autopsy series have shown that in cases of CUP, the lung is the most common site of primary followed by liver, pancreas and gastrointestinal tract [4]. Approximately 10-40\% of these patients have metastasis confined to the regional lymph nodes whereas in the rest, internal organs are involved [5]. In the former subgroup, cervical nodes are the most common nodal basins followed by axillary nodes. In general, treatment of CUP is guided by the suspected origin of the primary carcinoma and hence an extensive diagnostic work up is essential. CUP involving the inguinal nodes is rare accounting for only $1 \%$ to $3.5 \%$ of this subgroup of patients [6,7]. We are presenting a case of inguinal node Squamous cell carcinoma deposit of unknown primary site which was treated with surgery and chemotherapy.

\section{Case report}

A 35 year old premenopausal lady presented with history of right groin swelling of 3 month duration. On examination, she had $3 \mathrm{X} 2 \mathrm{~cm}$ nodal mass involving the right superficial inguinal nodes. Per vaginal and per rectal examination was within normal limits. FNAC of the nodal mass revealed presence of squamous cell carcinoma. Contrast enhanced computed tomography (CECT) of the abdomen and thorax revealed only right inguinal nodal mass but no other sites of disease. Papanicolaou test revealed normal cervix. In view of squamous histology in the inguinal nodes and no other site of primary detected, colposcopy and directed biopsy was done from the cervix. However there was no evidence of malignancy. In view of these, right inguinal block dissection was performed. Intra and post operative course was uneventful. Histopathological examination revealed one out of 12 nodes showing metastatic squamous cell carcinoma deposits with perinodal extension (Figure 1). On immunohistochemical examination, the deposits were positive for CK HMW, focally positive for p63 but negative for CK 7 and CK 20. Patient received 6 cycles of Paclitaxel and Carboplatin based chemotherapy as adjuvant treatment. Ideally this patient deserved adjuvant radiotherapy in view of perinodal extension, however as the primary may emerge at a later date either in cervix or in anal canal, radiotherapy was kept as a reserve treatment and only adjuvant chemotherapy was given. At 3 months follow up, patient was healthy and disease free. 


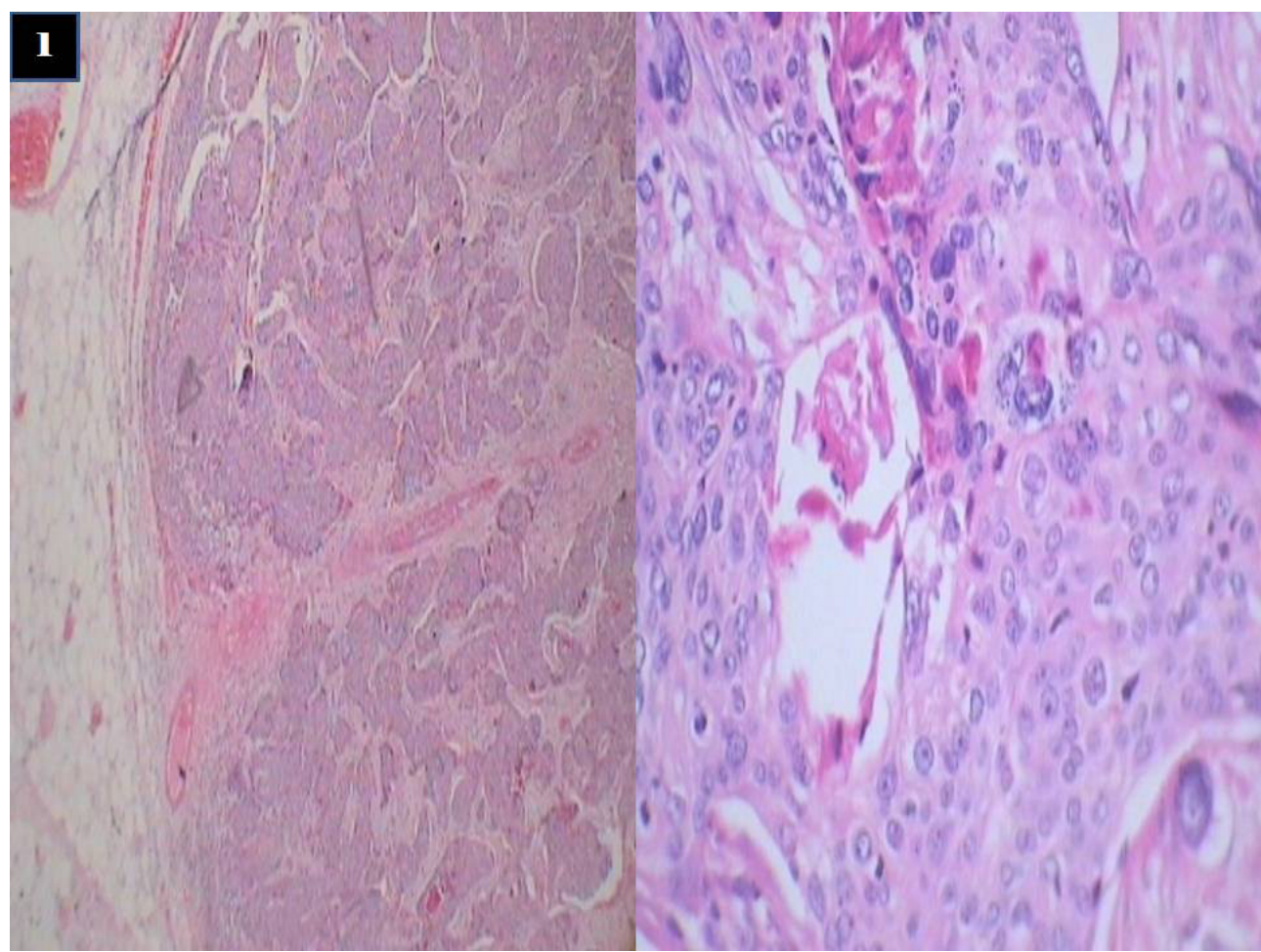

Figure 1: Photomicrograph showing nodal tissue with squamous cell carcinoma deposits

\section{Discussion}

CUP is defined as metastatic deposits for which no site of origin could be detected even after performing all the possible tests. It accounts for 3-5\% of all the malignancies and is often termed as 'an orphan disease' [1-3]. Although exact aetiology of CUP is unknown, several theories have been proposed. A concurrent but unrecognized primary cancer, a spontaneously regressed primary carcinoma that was either misdiagnosed or ignored are some of the explanations. Over last decade, there has been decrease in the incidence of CUP in many registries probably because of the evolution of new detection methods [2]. Peak age of incidence is 70 years and is equally common among males and females [8]. The present patient was middle aged lady presenting with groin nodal squamous cell carcinoma deposits with unknown primary.

Tobacco abuse and low socioeconomic status are considered as high risk factors for the development of CUP. Interestingly, a Nordic occupational cancer study found that doctors have the lowest risk $[9,10]$. A recent Swedish study found a well defined familial clustering of the cases of CUP [11]. Histologically, CUP are categorized into four major subtypes: well or moderately differentiated adenocarcinomas, squamous cell carcinoma, poorly differentiated adenocarcinomas and undifferentiated neoplasms. Among these, well or moderately differentiated adenocarcinomas account for more than half of the cases. In general, squamous cell carcinomas account for 15\% of all cases of CUP [12]. Although, Immunohistochemical examination (IHC) of the histopathology frequently can detect the primary cancer, it is labour intensive and costly. For present patient although IHC were done on the biopsy specimen, primary site could not be detected.

Diagnostic work up of cases of CUP includes a whole battery of laboratory tests in addition to detailed history and clinical examination. Need for Papanicolaou test and per vaginal examination in females, detailed examination of penis in males and per rectal examination in both sexes can't be over emphasized. Positron emission tomography (PET) scan has been found to be a very useful modality to detect the primary site particularly for cervical nodal CUP. Despite the development of newer diagnostic methods, the optimal algorithm in these rare cases remains to be established. In the present patient, CECT of the abdomen and thorax were done as PET CT was not available. We feel minimum tests required for cases of groin node SCC with unknown primary are PET CT, biopsy of the node with IHC and pap smear sos colposcopy.

Prognosis is dismal in spite of development of newer chemotherapeutic agents. However, there is a definite subgroup in this heterogeneous group of patients who have a favourable long term outcome. In particular, the prognosis is favourable in CUP confined to lymph nodes and with histology other than adenocarcinoma. In contrast, presence of liver metastasis or involvement of multiple viscera carries unfavourable prognosis [13-15]. Perinodal extension is the other important histological feature which carries worst prognosis as shown in a recent meta-analysis [16]. Hemminki et al reviewed the prognosis of patients with CUP and found that the location of the affected lymph nodes had a major effect on survival [17]. Intrapelvic and intra-abdominal lymph node metastases were most fatal and those in the head and neck were least fatal. The lowest hazard ratio (HR) for any site/histology type was for squamous cell carcinoma of the inguinal region. 
Inguinal nodes drain following areas: perineum, anal canal, lower portions of abdomen, back and the lower limbs in both sexes; vulva, vagina and cervix in females; penis in males. Hence enlargement of these nodes is because of the inflammation or neoplasm arising from one of these sub sites. Squamous cell carcinoma is the most common histology involving most of these sub sites. Hence squamous cell carcinoma deposits in the inguinal nodes with unknown primary can arise from any of these sub sites. Unfortunately IHCs rarely narrow the differential diagnosis in these cases. Surgery remains the mainstay of therapy in these cases with favourable prognosis. Use of adjuvant radiotherapy needs detailed discussion in the multidisciplinary meetings as re irradiation for the new primary that may emerge during follow up may not be feasible.

\section{Conclusion}

The purpose of presenting the present case was to stress the rarity of squamous cell carcinoma deposits in the inguinal nodes with unknown primary as well as the unique diagnostic and therapeutic dilemma they offer for the treating oncologists.

\section{References}

1. Pavlidis N, Fizazi K (2009) Carcinoma of unknown primary (CUP). Crit Rev Oncol Hematol 69: 271-8.

2. Randén M, Rutqvist LE, Johansson H (2009) Cancer patients without a known primary: incidence and survival trends in Sweden 1960-2007. Acta Oncol 48: 915-20.

3. van de Wouw AJ, Janssen-Heijnen ML, Coebergh JW, Hillen HF (2002) Epidemiology of unknown primary tumours; incidence and population-based survival of 1285 patients in Southeast Netherlands, 1984-1992. Eur J Cancer 38: 409-13.

4. Freudenberg LS, Rosenbaum-Krumme SJ, Bockisch A, Eberhardt W, Frilling A (2008) Cancer of unknown primary. Recent Results Cancer Res 170: 193-202.

5. Abbruzzese JL, Abbruzzese MC, Hess KR, Raber MN, Lenzi R, et al. (1994) Unknown primary carcinoma: natural history and prognostic factors in 657 consecutive patients. J Clin Oncol 12: 1272-80.

6. Greager JA, Wood D, Das Gupta TK (1983) Metastatic cancer from an undetermined primary site. J Surg Oncol 23: 73-6.

7. Didolkar MS, Fanous N, Elias EG, Moore RH (1977) Metastatic carcinomas from occult primary tumors. A study of 254 patients. Ann Surg 186: 625-30.

8. Centre for Epidemiology: Cancer incidence in Sweden 2005. Stockholm, Sweden, National Board of Health and Welfare, 2007.

9. Luke C, Koczwara B, Karapetis C, Pittman K, Price T, et al. (2008) Exploring the epidemiological characteristics of cancers of unknown primary site in an Australian population: implications for research and clinical care. Aust N Z J Public Health 32: 383-9.

10. Pukkala E, Martinsen JI, Lynge E, Gunnarsdottir HK, Sparén P, et al. (2009) Occupation and cancer - follow-up of 15 million people in five Nordic countries. Acta Oncol 48: 646-790.

11. Hemminki K, Ji J, Sundquist J, Shu X (2011) Familial risks in cancer of unknown primary: tracking the primary sites. J Clin Oncol 29: 435-40.

12. Petrović D, Muzikravić L, Jovanović D (2007) [Metastases of unknown origin--principles of diagnosis and treatment]. Med Pregl 60: 29-36.

13. Culine S (2009) Prognostic factors in unknown primary cancer. Semin Oncol 36: 60-4.

14. Pavlidis N, Briasoulis E, Hainsworth J, Greco FA (2003) Diagnostic and therapeutic management of cancer of an unknown primary. Eur J Cancer 39 : $1990-2005$.

15. Pavlidis N, Briasoulis E, Pentheroudakis G, On behalf of the ESMO Guidelines Working Group (2010) Cancers of unknown primary site: ESMO Clinical Practice Guidelines for diagnosis, treatment and follow-up. Ann Oncol 5: v228-31.

16. Luchini C, Nottegar A, Solmi M, Sergi G, Manzato E, et al. (2016) Prognostic implications of extranodal extension in node-positive squamous cell carcinoma of the vulva: A systematic review and meta-analysis. Surg Oncol 25: 60-5.

17. Hemminki K, Bevier M, Hemminki A, Sundquist J (2012) Survival in cancer of unknown primary site: population-based analysis by site and histology. Ann Oncol, doi: 10.1093/annonc/mdr536.

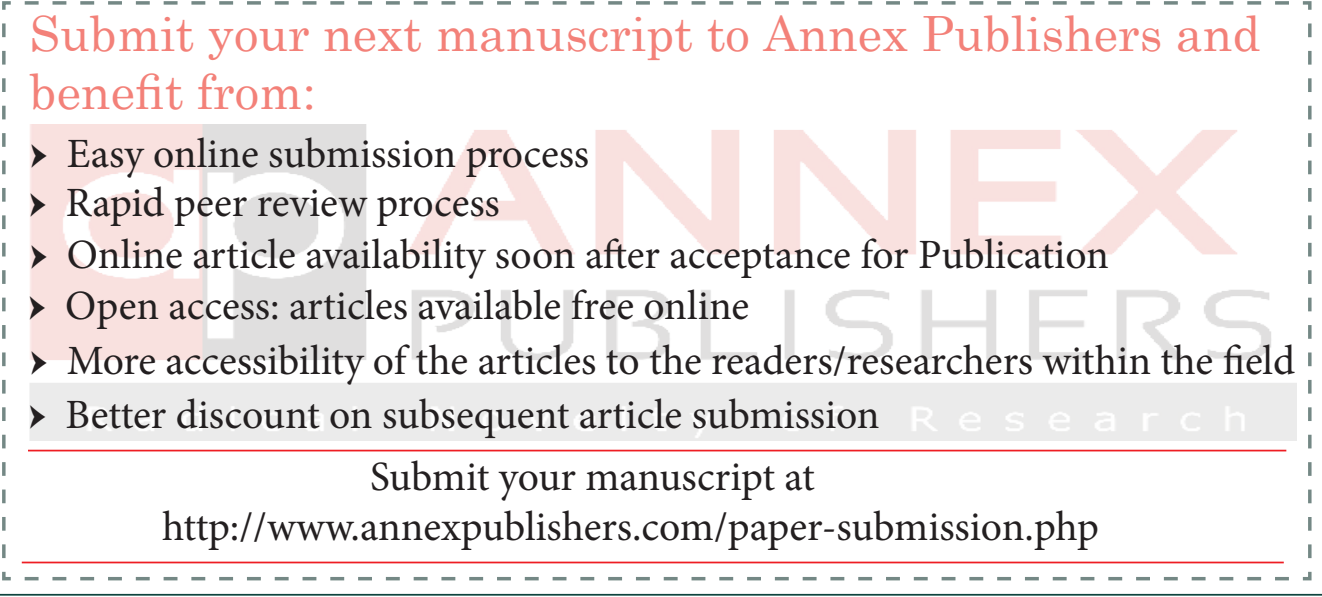

